\title{
Charge self-consistency in density functional theory combined with dynamical mean field theory: $k$-space reoccupation and orbital order
}

\author{
Sumanta Bhandary, ${ }^{1, *}$ Elias Assmann, ${ }^{1,2}$ Markus Aichhorn, ${ }^{2}$ and Karsten Held ${ }^{1}$ \\ ${ }^{1}$ Institute of Solid State Physics, TU Wien, 1040 Wien, Austria \\ ${ }^{2}$ Institute of Theoretical and Computational Physics, Graz University of Technology, Petersgasse 16, 8010 Graz, Austria
}

(Received 2 May 2016; revised manuscript received 21 September 2016; published 19 October 2016)

\begin{abstract}
We study the effects of charge self-consistency within the combination of density functional theory (DFT; WIEN2K) with dynamical mean field theory (DMFT; W2DYNAMICS) in a basis of maximally localized Wannier orbitals. Using the example of two cuprates, we demonstrate that even if there is only a single Wannier orbital with fixed filling, a noteworthy charge redistribution can occur. This effect stems from a reoccupation of the Wannier orbital in k-space when going from the single, metallic DFT band to the split, insulating Hubbard bands of DMFT. We analyze another charge self-consistency effect beyond moving charge from one site to another: the correlation-enhanced orbital polarization in a freestanding layer of $\mathrm{SrVO}_{3}$.
\end{abstract}

DOI: 10.1103/PhysRevB.94.155131

\section{INTRODUCTION}

Density functional theory (DFT) [1,2] is highly successful in predicting various material properties, such as crystal structures, ionization energies, and electrical, magnetic, and vibrational properties. Indeed, DFT is the de facto standard for calculating the physical properties of materials. But even the best approximations for the DFT exchange-correlation functional fail to describe one class of materials, known as strongly correlated systems. In these materials, the interaction between electrons is insufficiently screened to be amenable to the available functionals. One might add a static Coulomb correction within the so-called DFT+U formalism [3]. This often yields an improved description, in particular of strongly correlated insulators, but it has its own limitations: DFT $+\mathrm{U}$ is essentially a Hartree-Fock-like treatment with a single-Slaterdeterminant ground state. In this situation, the energy cost of the Coulomb interaction can only be avoided by symmetry breaking, which is hence largely overestimated.

Dynamic, albeit local, correlations can be taken into account by dynamical mean field theory (DMFT) [4-6], which has been merged with DFT for realistic calculations of correlated materials [7-10]. Here electrons can stay on or leave lattice sites dynamically so as to greatly suppress double occupation and the cost of the Coulomb interaction, even in a paramagnetic phase without any symmetry breaking. If one has a three-dimensional material at elevated temperatures, say room temperature, and if there is no magnetic or other phase transition close by [11], these local DMFT correlations prevail. Already the first applications showed that DFT+DMFT well describes transition metals [8], their oxides [12], and $f$-electron systems $[13,14]$.

In these early papers, so-called one-shot DFT+DMFT was used. That is, following a DFT calculation, the relevant correlated orbitals and the corresponding single-particle Hamiltonian were identified. This DFT Hamiltonian was supplemented by local Coulomb interactions for the $d$ - or $f$-orbitals and solved with DMFT. Physical properties such as the spectral function, susceptibility, or magnetization were calculated from this "one-shot" DMFT solution.

*bhandary@ifp.tuwien.ac.at
Since the DMFT correlations change the site and orbital occupation and consequently the charge density, a natural next step is to do a "charge self-consistent" (CSC) DFT+DMFT calculation [15-22]. That is, from the DMFT Green's function, a new charge distribution is calculated, which in turn serves as input for the DFT potential. This leads to a new DFT KohnSham Hamiltonian, and subsequently a new DMFT Green's function, etc. This cycle is repeated until convergence. While it has been pointed out in the literature [15-22] how the DMFT spectral function, the double counting, and the $d(f)$ energy level changes due to CSC for specific materials where charge is moved from one site to another, little attention has been paid to the redistributed charge itself, its spatial arrangement, and more.

The aforementioned change of double counting and $d$-level shift can be understood as follows: In a typical situation, say for a transition-metal oxide, the dominant $d$ states crossing the Fermi energy have some oxygen $p$ admixture; conversely, the oxygen states below the Fermi level have some $d$ contribution. Including electronic correlations in a so called $d+p$ DMFT calculation will reduce the $d$ occupation somewhat, and increase the $p$ occupation on the oxygen sites. In the next DFT step, the larger $p$ occupation will increase the $p$ (Hartree) energy and decrease the $d$ (Hartree) energy. This counteracts the first shot DMFT to have fewer $d$ and more $p$ electrons, dampening the charge redistribution of the "first-shot" DFT+DMFT.

In this paper, we study the effects of CSC beyond this gross effect of a $p$ - $d$ orbital and site reoccupation. In Sec. II, we recapitulate the CSC DFT+DMFT approach and outline our implementation thereof. In Secs. III A and III B, we show that even in a single-orbital, $d$-only DMFT calculation, there is a charge redistribution akin the $d$-p reoccupation effect mentioned above. This runs counter to the naive expectation that there can be no charge redistribution in this situation since the number of electrons in the single, predominately $d$-like orbital centered around the transition-metal site is fixed. The two materials studied, where a restriction to a single $d$ band is justified, are $\mathrm{Sr}_{2} \mathrm{CuTeO}_{6}$ and $\mathrm{HgBa}_{2} \mathrm{CuO}_{4}$ (Secs. III A and III B, respectively). In Sec. III C, we study the effect of correlation-induced orbital order on the charge redistribution and self-consistent DFT+DMFT results. Specifically, we 
consider an ultrathin layer of the cubic perovskite material $\mathrm{SrVO}_{3}$, where breaking of the cubic symmetry stabilizes the in-plane $x y$ orbital against the $x z$ and $y z$ orbitals. This orbital ordering is strongly enhanced in DMFT because of electronic correlations. Finally, Sec. IV summarizes our main findings.

\section{METHODOLOGY}

We now present the formalism and our implementation of CSC DFT+DMFT, which is in a basis of maximally localized Wannier functions (MLWFs). For these, the measure of localization introduced by Marzari and Vanderbilt [23] is the spread in real space. This allows for a very flexible approach that can be adapted to any band-structure method. Moreover, MLWFs allow more general initial projections, e.g.,, bond-centered orbitals. They are also more well-defined through the localization procedure. Our starting point is the WIEN2WANNIER [24] interface between WIEN2K [25] and WANNIER90 [26], and the W2DYNAMICS [27] continuous-time quantum Monte Carlo [28] DMFT implementation.

We combine and extend these methods to include CSC. For the sake of completeness, and given the sparse presentation in the literature, we will recapitulate here the CSC DFT+DMFT approach and discuss the peculiarities of our implementation. Readers only interested in the physical applications and effects of CSC can safely skip the rest of this section.

The CSC DFT+DMFT method relies on the simultaneous convergence of two local observables: the electronic density as the central quantity of DFT, and the local Green's function as the central quantity of DMFT. Both mutually affect each other in the CSC cycle. The charge density at position $\mathbf{r}$ is given by

$$
\rho(\mathbf{r})=\frac{1}{\beta} \sum_{n} G\left(\mathbf{r}, \mathbf{r} ; i \omega_{n}\right) e^{i \omega_{n} 0^{+}},
$$

while the local DMFT Green's function is

$$
G_{m m^{\prime}}\left(i \omega_{n}\right)=\int d \mathbf{r} d \mathbf{r}^{\prime} \chi_{m}^{*}(\mathbf{r}) \chi_{m^{\prime}}\left(\mathbf{r}^{\prime}\right) G\left(\mathbf{r}, \mathbf{r}^{\prime} ; i \omega_{n}\right)
$$

in the basis of localized Wannier orbitals $\chi_{m}$. Here, $m, m^{\prime}$ enumerate orbitals on a site, $\beta$ is the inverse temperature, and the factor $e^{i \omega_{n} 0^{+}}$ensures the convergence of the sum over Matsubara frequencies $\omega_{n}=(2 n+1) \pi / \beta$.

In both expressions, there appears the full Green's function of the solid, which can be written as

$$
G\left(\mathbf{r}, \mathbf{r}^{\prime} ; i \omega\right)=\left\langle\mathbf{r}\left|\left[i \omega_{n}+\mu+\frac{\nabla^{2}}{2}-\hat{V}_{\mathrm{KS}}-\Delta \hat{\Sigma}\right]^{-1}\right| \mathbf{r}^{\prime}\right\rangle,
$$

with $-\frac{\nabla^{2}}{2}, V_{\mathrm{KS}}$, and $\mu$ being the kinetic energy operator, Kohn-Sham (KS) effective potential, and chemical potential, respectively. The effective local self-energy $\Delta \hat{\Sigma}=\hat{\Sigma}-\hat{\Sigma}_{\mathrm{dc}}$ is determined from the DMFT self-energy $\hat{\Sigma}$ by subtracting a double-counting correction term $\hat{\Sigma}_{\mathrm{dc}}$, which, as far as possible, accounts for electronic correlations already included in DFT. In all our calculations, we have used fully localized limit (FLL) double counting. For the single-band cases, i.e., $\mathrm{HgBa}_{2} \mathrm{CuO}_{4}$ and $\mathrm{Sr}_{2} \mathrm{CuTeO}_{6}$, it plays no role except for shifting the chemical potential. The KS potential depends on $\mathbf{r}$ and consists of the external potential $V_{\text {ext }}$ due to the nuclei, a Hartree potential $V_{H}$ describing part of the electron-electron Coulomb repulsion, and an exchange-correlation potential $V_{\mathrm{xc}}$. The latter is obtained here within the generalized gradient approximation (GGA) [29], but other functionals are possible as well, e.g., hybrid functionals to improve on the exchange contribution.

In DFT, the effective potential is obtained from a charge self-consistent procedure, shown in the upper left part of Fig. 1. This DFT cycle starts with an initial choice for the electron density, from which the effective potential $V_{\mathrm{KS}}$ is

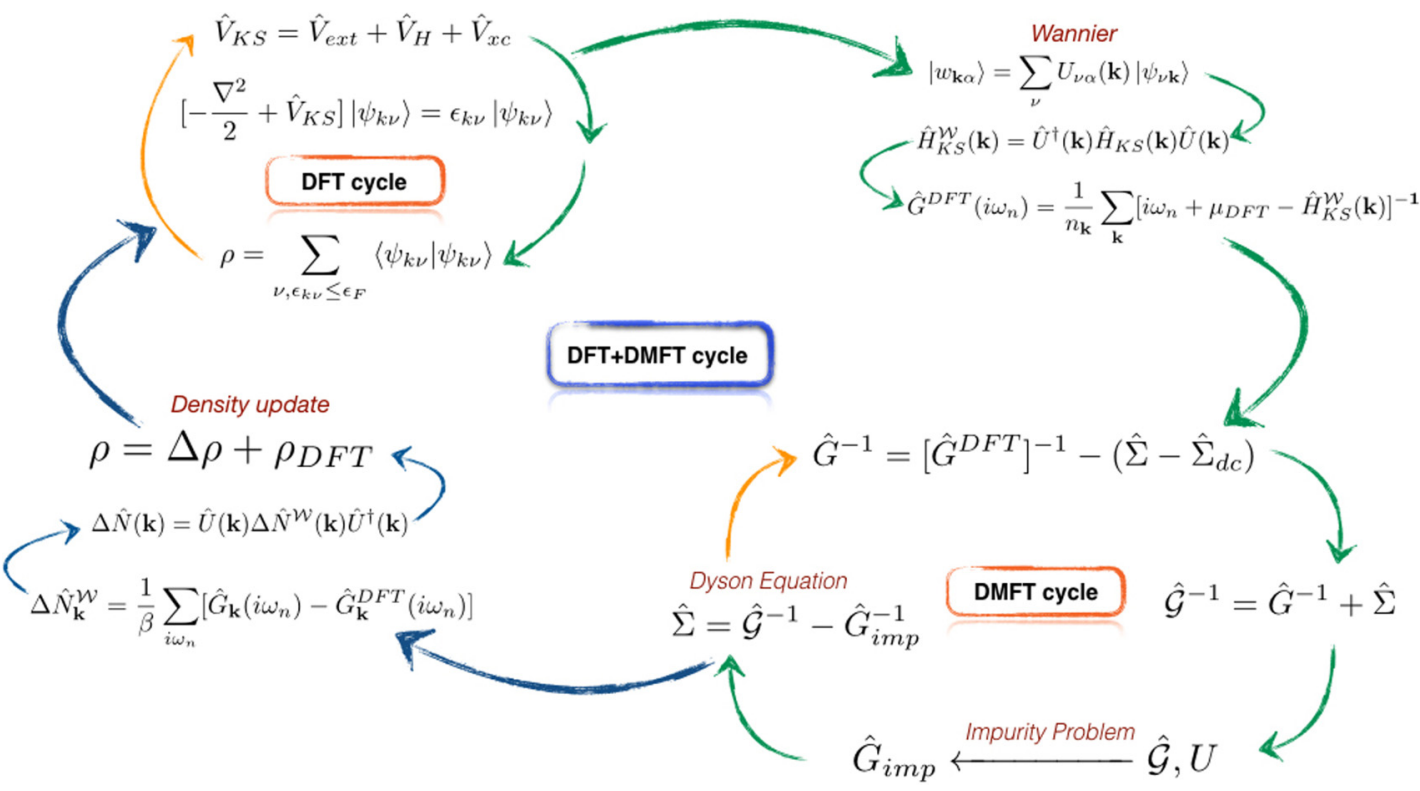

FIG. 1. Schematic representation of the DFT+DMFT approach. In a non-CSC or "one-shot" DFT+DMFT calculation, the step labeled "density update" is not performed; both the DFT and DMFT cycles are closed separately. By contrast, in CSC DFT+DMFT, neither DFT nor DMFT is iterated individually; instead, both of them are closed together. In terms of the schematic, a one-shot calculation follows the orange arrows, which close the individual cycles. A CSC calculation follows the blue arrows instead, which close the CSC cycle. (The green arrows are followed in either case.) 
constructed. Incorporating $V_{\mathrm{KS}}$, the Kohn-Sham equation is solved to obtain a new density, and so forth until convergence. The DFT cycle closes with a converged charge density and provides a reasonable electronic structure as a starting point for DMFT calculations.

There is, however, an important step between DFT and DMFT, identifying a localized basis (upper right part in Fig. 1) since DMFT treats only local correlations. To this end, we employ Wannier functions that are constructed by Fourier transform of the DFT Bloch waves $\left|\psi_{\nu \mathbf{k}}\right\rangle$ :

$$
\left|w_{\alpha \mathbf{R}}\right\rangle=\frac{\Omega}{(2 \pi)^{3}} \int_{\mathrm{BZ}} d \mathbf{k} e^{-i \mathbf{k} \cdot \mathbf{R}} \sum_{\nu=1}^{\mathcal{C}} U_{\nu \alpha}(\mathbf{k})\left|\psi_{\nu \mathbf{k}}\right\rangle .
$$

Here, $\hat{U}(\mathbf{k})$ is a unitary matrix, $\Omega$ denotes the volume of the unit cell, and $v$ and $\alpha$ are the band indices of the Bloch waves and Wannier functions, respectively. We assume here that we can restrict ourselves to a band window with only $\mathcal{C}$ Bloch waves. In the scheme of maximally localized Wannier functions [23], $\hat{U}(\mathbf{k})$ is obtained by minimizing the spread of the Wannier functions.

Equation (4) works for isolated bands. However, in most cases, the target bands are "entangled" with further bands at least at some k-points. These additional bands might be less important for the physics, but they need to be projected out by a so-called "disentanglement" procedure. At each kpoint, there is a set of $\mathcal{C}^{o}(\mathbf{k})$ Bloch functions that is larger than or equal to the number of target bands, i.e., $\mathcal{C}^{o}(\mathbf{k}) \geqslant \mathcal{C}$. The disentanglement transformation takes the form

$$
\left|w_{\alpha \mathbf{R}}\right\rangle=\frac{\Omega}{(2 \pi)^{3}} \int_{\mathrm{BZ}} d \mathbf{k} e^{-i k \mathbf{R}} \sum_{\nu^{\prime}=1}^{\mathcal{C}} \sum_{\nu=1}^{\mathcal{C}^{o}(\mathbf{k})} V_{\nu \nu^{\prime}}(\mathbf{k}) U_{\nu^{\prime} \alpha}(\mathbf{k})\left|\psi_{\nu \mathbf{k}}\right\rangle .
$$

Here, the band index $v$ belongs to the "outer window" with $\mathcal{C}^{o}(\mathbf{k})$ Bloch wave functions, while $v^{\prime}, \alpha$ label the $\mathcal{C}$ target bands. Hence, $\hat{V}(\mathbf{k})$ is a rectangular $\mathcal{C}^{o}(\mathbf{k}) \times \mathcal{C}$ matrix. A Fourier transformation of $\left|w_{\alpha \mathbf{R}}\right\rangle$ leads to the Wannier orbitals in $\mathbf{k}$-space whose occupation will be the focus of the physics discussed below:

$$
\left|w_{\alpha \mathbf{k}}\right\rangle=\sum_{\mathbf{R}} e^{i k \mathbf{R}}\left|w_{\alpha \mathbf{R}}\right\rangle=\sum_{\nu^{\prime} \nu} V_{\nu \nu^{\prime}}(\mathbf{k}) U_{\nu^{\prime} \alpha}(\mathbf{k})\left|\psi_{\nu \mathbf{k}}\right\rangle .
$$

The Hamiltonian in Wannier space, $\mathcal{W}$, is defined in terms of the $\left|w_{\alpha \mathbf{k}}\right\rangle$ and obtained by a unitary transformation for isolated bands and with an additional projection ("downfold") in the case of entangled bands, i.e.,

$$
\begin{gathered}
\hat{H}_{\mathrm{KS}}^{\mathcal{W}}(\mathbf{k})=\hat{U}^{\dagger}(\mathbf{k}) \hat{H}_{\mathrm{KS}}(\mathbf{k}) \hat{U}(\mathbf{k}), \\
\hat{H}_{\mathrm{KS}}^{\mathcal{W}}(\mathbf{k})=\hat{U}^{\dagger}(\mathbf{k}) \hat{V}^{\dagger}(\mathbf{k}) \hat{H}_{\mathrm{KS}}(\mathbf{k}) \hat{V}(\mathbf{k}) \hat{U}(\mathbf{k}),
\end{gathered}
$$

respectively.

In DMFT, this Hamiltonian is now supplemented with the local Coulomb interactions, and the lattice problem defined in this way is mapped onto an auxiliary impurity problem that is solved self-consistently [5,6]. The noninteracting Green's function $\hat{\mathcal{G}}\left(i \omega_{n}\right)$ of the impurity problem can be considered as a dynamical mean field. The DMFT algorithm (see the lower right part of Fig. 1) consists of the following: (i) Applying the lattice Dyson equation for the local interacting Green's function $\hat{G}\left(i \omega_{n}\right)$,

$$
\hat{G}\left(i \omega_{n}\right)=\frac{1}{n_{\mathbf{k}}} \sum_{\mathbf{k}}\left[i \omega_{n}+\mu-\hat{H}_{\mathrm{KS}}^{\mathcal{W}}(\mathbf{k})-\hat{\Sigma}+\hat{\Sigma}_{\mathrm{dc}}\right]^{-1} .
$$

To enhance convergence, one normally starts with $\hat{\Sigma}=\hat{\Sigma}_{\mathrm{dc}}$, i.e., using the Hartree energy as a first guess for the self-energy. A total number of $\mathbf{k}$-points, $n_{\mathbf{k}}$, is considered in the reducible Brillouin zone.

(ii) Applying the impurity Dyson equation, which relates the noninteracting impurity Green's function to the (lattice and impurity) self-energy and interacting Green's function,

$$
\hat{\mathcal{G}}\left(i \omega_{n}\right)^{-1}=\hat{\Sigma}\left(i \omega_{n}\right)+\left[\hat{G}\left(i \omega_{n}\right)\right]^{-1} .
$$

(iii) Solving the Anderson impurity problem (AIM) defined by the noninteracting Green's function and the local Coulomb interaction $U$, i.e., calculating its interacting Green's function $\hat{G}_{\mathrm{imp}}\left(i \omega_{n}\right)$,

$$
\hat{\mathcal{G}}\left(i \omega_{n}\right), U \stackrel{\text { AIM }}{\longrightarrow} \hat{G}_{\text {imp }}\left(i \omega_{n}\right) .
$$

This is numerically the most involved step; we employ the continuous-time quantum Monte-Carlo method [28] in the W2DYNAMICS implementation [27].

(iv) Applying the impurity Dyson equation once again, this time to calculate the self-energy as the difference between the inverse noninteracting impurity Green's function $\hat{\mathcal{G}}\left(i \omega_{n}\right)$ and the interacting (lattice and impurity) Green's function $\hat{G}\left(i \omega_{n}\right)$,

$$
\hat{\Sigma}\left(i \omega_{n}\right)=\hat{\mathcal{G}}^{-1}\left(i \omega_{n}\right)-\hat{G}_{\mathrm{imp}}^{-1}\left(i \omega_{n}\right) .
$$

This self-energy is now used again in step (i) to calculate a new local Green's function. This procedure is referred to as the "DMFT cycle" in Fig. 1. In a one-shot DFT+DMFT calculation, we would stop after this DMFT calculation, extracting the interacting Green's function and further physical quantities from the converged DMFT solution.

By contrast, in CSC DFT+DMFT calculations, we need to determine the DMFT-modified electron density (lower left segment in Fig. 1), recalculate from this the Kohn-Sham potential and the Bloch waves without DFT self-consistency, and redo for these a Wannier function projection, which is the starting point for another DMFT step (see the green and blue arrows in Fig. 1).

We still need to discuss how we calculate the DMFTmodified electron density $\rho(\mathbf{r})=\rho_{\mathrm{DFT}}(\mathbf{r})+\Delta \rho(\mathbf{r})$, which we defined in terms of the Kohn-Sham or DFT $\rho_{\mathrm{DFT}}(\mathbf{r})$ and the correlation-induced difference $\Delta \rho(\mathbf{r})$. The latter can be calculated as [18]

$$
\begin{aligned}
\Delta \rho(\mathbf{r}) & =\rho_{\mathrm{DMFT}}(\mathbf{r})-\rho_{\mathrm{DFT}}(\mathbf{r}) \\
& =\left\langle\mathbf{r}\left|\left(\hat{G}-\hat{G}^{\mathrm{DFT}}\right)\right| \mathbf{r}\right\rangle \\
& \left.=\langle\mathbf{r}| \hat{G}^{\mathrm{DFT}}\left[\Delta \hat{\Sigma}+\left(\mu_{\mathrm{DFT}}-\mu\right)\right] \hat{G}\right]|\mathbf{r}\rangle,
\end{aligned}
$$

where $\mu_{\mathrm{DFT}}$ and $\mu$ are the DFT and DMFT chemical potentials, respectively, and $\hat{G}^{\mathrm{DFT}}\left(i \omega_{n}\right)=\sum_{\mathbf{k}}\left[i \omega_{n}+\mu_{\mathrm{DFT}}-\hat{H}_{\mathrm{KS}}^{\mathcal{W}}(\mathbf{k})\right]^{-1}$ is the DFT Green's function. It is computationally convenient to express $\Delta \rho(\mathbf{r})$ in momentum space, which can be deduced 
from Eq. (13) as

$$
\begin{aligned}
\Delta \rho(\mathbf{r})= & \frac{1}{n_{\mathbf{k}}} \sum_{\mathbf{k}, \alpha \alpha^{\prime}}\left\langle\mathbf{r} \mid w_{\alpha \mathbf{k}}\right\rangle \Delta N_{\alpha \alpha^{\prime}}^{\mathcal{W}}(\mathbf{k})\left\langle w_{\alpha^{\prime} \mathbf{k}} \mid \mathbf{r}\right\rangle, \\
\Delta \hat{N}^{\mathcal{W}}(\mathbf{k})= & \frac{1}{\beta} \sum_{n} \hat{G}^{\mathrm{DFT}}\left(\mathbf{k}, i \omega_{n}\right)\left[\Delta \hat{\Sigma}\left(i \omega_{n}\right)+\Delta \mu\right] \\
& \times \hat{G}\left(\mathbf{k}, i \omega_{n}\right)
\end{aligned}
$$

with $\Delta \hat{\Sigma}=\hat{\Sigma}-\hat{\Sigma}_{\mathrm{dc}}$ and $\Delta \mu=\mu_{\mathrm{DFT}}-\mu$. It should be noted that no convergence factor in the frequency summation needs to be used for $\Delta \hat{N}^{\mathcal{W}}(\mathbf{k})$ because both Green's functions decay asymptotically as $1 / \omega_{n}$. Note that the change of occupation in Wannier space, $\Delta N_{\alpha, \alpha^{\prime}}^{\mathcal{W}}$, has an explicit k-dependence, which will have significant consequences in the following section.

To update the DFT charge density, we need to transform $\Delta \hat{N}^{\mathcal{W}}(\mathbf{k})$ from the Wannier to the Bloch basis using the unitary and disentanglement matrices, $\hat{U}(\mathbf{k})$ and $\hat{V}(\mathbf{k})$, that define this transformation:

$$
\begin{gathered}
\Delta \hat{N}(\mathbf{k})=\hat{U}(\mathbf{k}) \Delta \hat{N}^{\mathcal{W}}(\mathbf{k}) \hat{U}^{\dagger}(\mathbf{k}) \\
\Delta \hat{N}(\mathbf{k})=\hat{V}(\mathbf{k}) \hat{U}(\mathbf{k}) \Delta \hat{N}^{\mathcal{W}}(\mathbf{k}) \hat{U}^{\dagger}(\mathbf{k}) \hat{V}^{\dagger}(\mathbf{k})
\end{gathered}
$$

Knowing the correlation-induced change of occupation in the Bloch or Kohn-Sham basis, we can finally calculate the modified density since we know the spatial density $D_{v^{\prime} v}^{\mathbf{k}}(\mathbf{r})=$ $\psi_{\mathbf{k} v}(\mathbf{r}) \psi_{\mathbf{k} v^{\prime}}^{*}(\mathbf{r})$ of each Bloch wave:

$$
\Delta \rho(\mathbf{r})=\frac{1}{n_{\mathbf{k}}} \sum_{\mathbf{k}} \sum_{\nu \nu^{\prime}=1}^{\mathcal{C}^{o}} D_{\nu^{\prime} v}^{\mathbf{k}}(\mathbf{r}) \Delta N_{\nu \nu^{\prime}}(\mathbf{k}) .
$$

The full CSC DFT+DMFT hence consists of the following work flow, schematically depicted in Fig. 1:

(i) A converged charge density is obtained within DFT to have a reasonable electronic structure to start with (upper left part of Fig. 1). The target bands are identified as a prelude for the Wannier projection. In the following CSC DMFT cycle (green and blue arrows in Fig. 1), a single DFT iteration is performed to update the DFT Kohn-Sham Hamiltonian (i.e., without the orange arrow in the upper left part). We employ the WIEN2K program package here.

(ii) Maximally localized Wannier functions are computed within the target subspace as explained in Eqs. (4)-(6) (upper right section of Fig. 1). The DFT Kohn-Sham Hamiltonian is transformed into the Wannier basis following Eq. (7). We employ WIEN2WANNIER [24] and WANNIER90 [26] to this end.

(iii) A single DMFT cycle is performed using W2DYNAMICS [27] (lower right part of Fig. 1). This provides the self-energy $\hat{\Sigma}$, local Green's function $\hat{G}$, and the DMFT chemical potential $\mu$, which is fixed to the particle number. Let us note that, for practical purposes, it is beneficial to start with a converged "one-shot" DFT + DMFT calculation. Moreover, a mixing (underrelaxation) between old and new DMFT self-energy is employed.

(iv) For the correlated charge distribution (lower left part of Fig. 1), first $\Delta N^{\mathcal{W}}(\mathbf{k})$ is calculated taking the difference between DMFT and DFT Green's functions, $\hat{G}$ and $\hat{G}_{\mathrm{DFT}}$, as in Eq. (14). As described in Eqs. (16)-(18), $\Delta N^{\mathcal{W}}(\mathbf{k})$ is transformed back to the DFT eigenbasis and used to obtain the correlation-induced change of density $\Delta \rho(\mathbf{r})$ and the total density $\rho(\mathbf{r})$ of the correlated solution.

(v) The DFT+DMFT charge density, $\rho(\mathbf{r})$, is finally compared with the old density. If the difference does not satisfy the convergence criterion (i.e., the WIEN2K charge convergence criterion with a value of $1 \times 10^{-4} e$ ), the new density is mixed with the old density and the result serves as the new density for a new $V_{\mathrm{DFT}}$ and a new solution of the Kohn-Sham equation, etc., until convergence. At the same time, a convergence of $\hat{G}(\tau)$ is also checked.

\section{APPLICATIONS}

In the following, fully CSC DFT+DMFT calculations are employed to shed light on correlation-induced charge redistribution beyond the gross effect of moving electrons from a WF centered at one atom to a WF centered at another atom. Two cuprates, $\mathrm{Sr}_{2} \mathrm{CuTeO}_{6}$ and $\mathrm{HgBa}_{2} \mathrm{CuO}_{4}$, whose physics is dominated by a single band, are studied. The systems are different in several aspects. First $\mathrm{Sr}_{2} \mathrm{CuTeO}_{6}$ exhibits a single isolated band around the Fermi energy, while in $\mathrm{HgBa}_{2} \mathrm{CuO}_{4}$ the single $d$-band is entangled with other bands crossing it. On the technical side, this requires disentanglement to project onto a single Wannier $d$ orbital for $\mathrm{HgBa}_{2} \mathrm{CuO}_{4}$, as discussed in the previous section.

Next, a multiorbital situation is considered with a single, free-standing layer of $\mathrm{SrVO}_{3}$ and $t_{2 g}$ orbitals at the Fermi energy that are well isolated from the other orbitals. Here, the interplay between structural confinement, orbital ordering, electronic correlations, and CSC is discussed in detail.

\section{A. $\mathrm{Sr}_{2} \mathrm{CuTeO}_{6}$}

To describe the physics of cuprates, an effective singleband model can be derived where the contributing orbital is predominantly of $\mathrm{Cu} d_{x^{2}-y^{2}}$ character, with some admixture of $\mathrm{O} p_{x / y}$. The compound, $\mathrm{Sr}_{2} \mathrm{CuTeO}_{6}$, exhibits squarelattice Heisenberg antiferromagnetism [30] in a quasi-twodimensional plane, consisting of $\mathrm{Cu}$ and $\mathrm{O}$ atoms; see Fig. 2 (left). It is quite unique in the cuprate group that it has a completely isolated and weakly dispersing band around the Fermi energy; see the white band in Fig. 2 (right). Thus, no disentanglement is needed in this material.

We take for our calculations the $I 4 / \mathrm{m}$ symmetry of the lattice with the experimental lattice parameters [31], i.e., inplane lattice constant $a=5.4308 \AA$ and out-of-plane lattice constant $c=8.4664 \AA$. A slight complication of the lattice structure is that the $\mathrm{CuO}_{6}$ octahedra in $\mathrm{Sr}_{2} \mathrm{CuTeO}_{6}$ are rotated around the $z$-direction. In contrast to the $\mathrm{CuO}_{2}$ planes of other cuprates, cf. Sec. III B, $\mathrm{Sr}_{2} \mathrm{CuTeO}_{6}$ has planes with four O per $\mathrm{Cu}$; no oxygen is shared, which explains the low itinerancy.

In DFT, $\mathrm{Sr}_{2} \mathrm{CuTeO}_{6}$ is a metal with a single half-filled band crossing the Fermi energy, predominantly of $\mathrm{Cu} d_{x^{2}-y^{2}}$ character. Electronic correlations result, however, in an insulating phase with two Hubbard bands separated by $U$. This is captured in our DMFT calculations, performed with $U=6.5 \mathrm{eV}$ at inverse temperature $\beta=40 \mathrm{eV}$. During the charge self-consistency cycle, the self-energy and density are underrelaxed; $1000 k$-points are considered in the reducible 


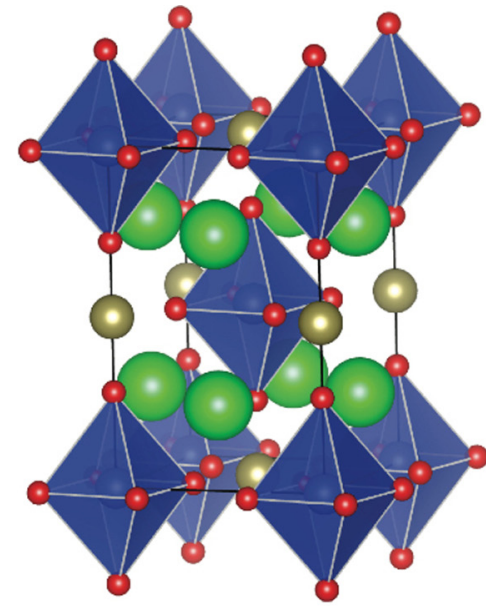

(a)

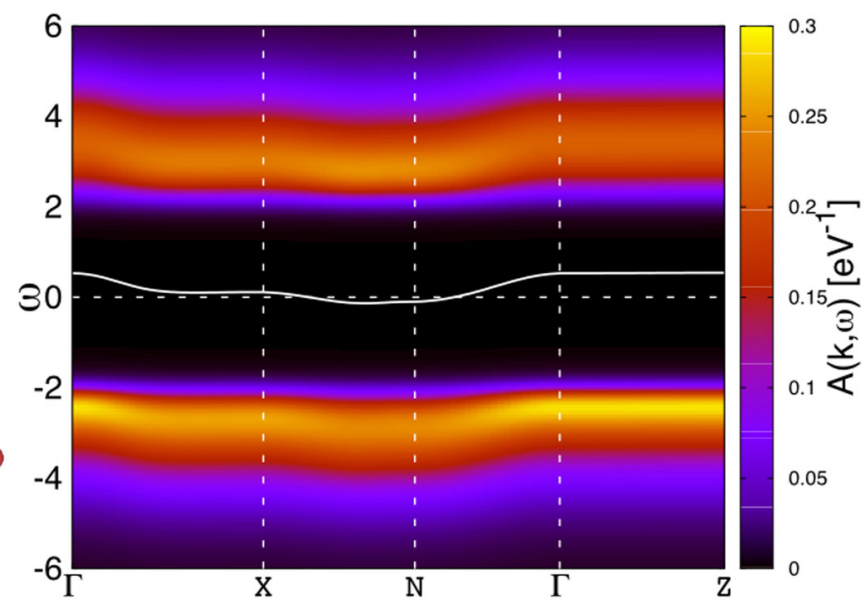

(b)

FIG. 2. (a) Crystal structure of $\mathrm{Sr}_{2} \mathrm{CuTeO}_{6}$ with green, golden, and red balls representing $\mathrm{Sr}$, $\mathrm{Te}$, and $\mathrm{O}$, respectively; the Cu sites are in the center of the blue octahedra that are elongates along the $c$-axis. (b) Spectral function, $A(\mathbf{k}, w)$, as calculated by DFT + DMFT along a high-symmetry path through the Brillouin zone. The white curve depicts a well-separated Wannier band; the Fermi energy is set at zero.

Brillouin zone for all the calculations. The half-filled DFT band remains half-filled in DMFT.

Naively one might expect that for an unchanged $d$-electron occupation (half-filling) there can be no CSC effect. However, the occupation in k-space is altered. In DFT (white band in Fig. 2), some k-points (in between $X-N-\Gamma$ ) are below the Fermi level, and hence are filled with one electron, whereas for all other $\mathbf{k}$-points the occupation is zero.

In DMFT this half-filled band is split into two Hubbard bands that are broadened because of the imaginary part of the self-energy, namely the lifetime. This splitting means that now every k-point is occupied with half an electron (lower Hubbard band), whereas the remaining half electronic state (upper Hubbard band) remains unoccupied. That is, we have a major change of the occupation $\Delta N(\mathbf{k})$ in $\mathbf{k}$-space, as calculated from the differences between $G$ and $G_{\mathrm{DFT}}$ at each k-point in Eq. (14). For the orbital occupation, the sum over the entire Brillouin zone is taken, preserving the number of electrons in the $d_{x^{2}-y^{2}}$ orbital.

For the change of charge $\Delta \rho(\mathbf{r})$ in real space, however, each $\Delta N(\mathbf{k})$ in Eq. (18) is weighted with the spatial distribution of the corresponding Wannier functions. Hence, the splitting into Hubbard bands results in a charge redistribution: the Wannier functions have a different spatial dependence at each $\mathbf{k}$-point.

This correlation-induced correction to the charge density within the $\mathrm{Cu}-\mathrm{O}$ plane is shown in Fig. 3. Here, the yellow (cyan) color corresponds to a gain (loss) of electron density in real space. As the single band of our consideration is predominantly of $d_{x^{2}-y^{2}}$ character, the contribution of each sign has the same orbital symmetry; the total change in density within the unit cell (shown as the black dashed box) is zero. The charge redistribution around each $\mathrm{Cu}$ ion can be understood easily for cubic crystal symmetry; see the discussion on $\mathrm{HgBa}_{2} \mathrm{CuO}_{4}$ in Sec. III B. Here, with lower symmetry, the charge redistribution around each $\mathrm{Cu}$ ion shows eight lobes with positive and negative contributions. Each $\mathrm{Cu}$ is surrounded by four oxygen atoms at the edges of the dotted box. As one can clearly see, the positive contribution at these $\mathrm{O}$ sites is larger than the negative one. That means that even in our $d$-only model calculation, there is some charge redistribution from $\mathrm{Cu} d$ to oxygen $p$. This is akin to the situation in $d$ - $p$ models where charge is moved from $d$ to $p$ orbitals as well. However, in our calculation this effect occurs even though we have only a single orbital in the DMFT calculation. This Wannier orbital is centered around the $\mathrm{Cu}$ sites and is predominantly of $d_{x^{2}-y^{2}}$ character. But it has some admixture of oxygen $p$, i.e., it has some charge density at the neighboring oxygen sites as well. This admixture requires some k-dependence of the Wannier functions [Eq. (6)], and the occupation is reduced, e.g., around the $N$ point, while it is increased in the remainder, eventually leading to the charge distribution pattern of Fig. 3.

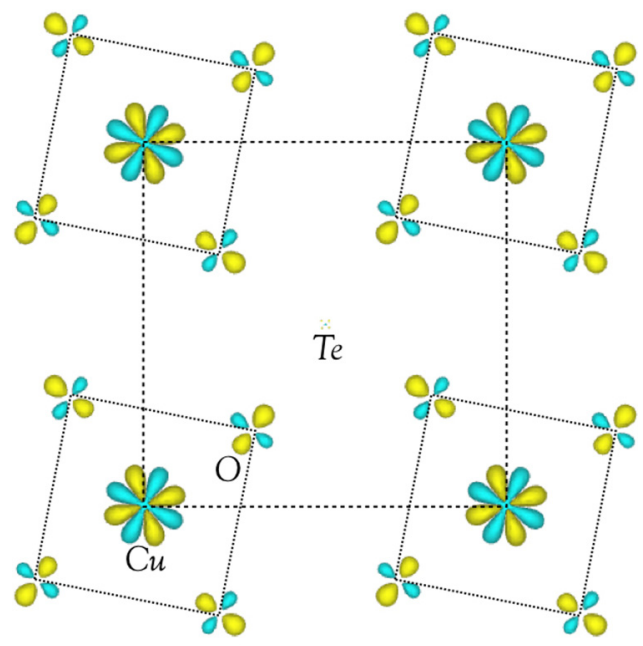

FIG. 3. Isosurface plot of the correlation-induced charge-density difference, $\Delta \rho(\mathbf{r})=\rho_{\text {DMFT }}(\mathbf{r})-\rho_{\mathrm{DFT}}(\mathbf{r})$, in $\mathrm{Sr}_{2} \mathrm{CuTeO}_{6}$. Yellow and cyan correspond to positive and negative $\Delta \rho(\mathbf{r})$ 's at an isovalue of $4 \times$ $10^{-3}$ electrons $/$ bohr $^{3}$; the dashed and dotted lines represent the unit cell and the (rotated) four $\mathrm{O}$ atoms around each $\mathrm{Cu}$ site, respectively. 


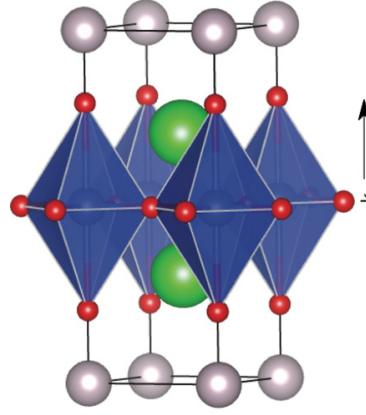

(a)

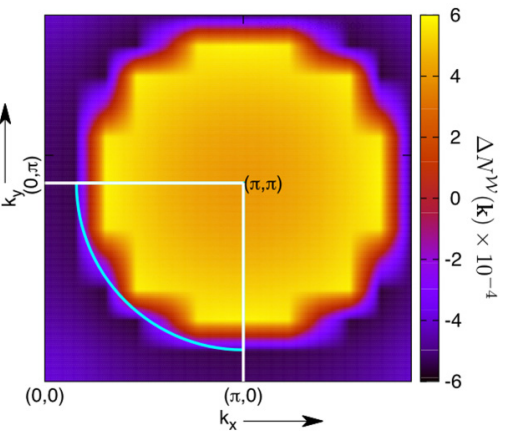

(b)
FIG. 4. (a) Crystal structure of $\mathrm{HgBa}_{2} \mathrm{CuO}_{4}$. Green, pink, blue, and red balls represent $\mathrm{Ba}, \mathrm{Hg}, \mathrm{Cu}$, and $\mathrm{O}$ atoms, respectively. (b) k-dependence of $\Delta N^{\mathcal{W}}$, i.e., the change of occupation of the Wannier orbitals in $\mathbf{k}$-space for the $k_{z}=0$ plane in the Brillouin zone. The high-symmetry points $\Gamma(0,0), X(\pi, 0)$, and $M(\pi, \pi)$ are marked. The cyan curve depicts the DFT Fermi surface, which separates positive (yellow) and negative (blue) $\Delta N^{\mathcal{W}}$ contributions.

\section{B. $\mathrm{HgBa}_{2} \mathrm{CuO}_{4}$}

Let us now turn to $\mathrm{HgBa}_{2} \mathrm{CuO}_{4}$, a prototype of the hightemperature cuprate superconductors [32]. The arrangement of the $\mathrm{CuO}_{6}$ octahedra is distinctly different in undoped $\mathrm{HgBa}_{2} \mathrm{CuO}_{4}$ compared to that of $\mathrm{Sr}_{2} \mathrm{CuTeO}_{6}$; see the crystal structure in Fig. 4(a). The system belongs to the space group $P 4 / m m m$, with $\mathrm{Hg}, \mathrm{BaO}, \mathrm{CuO}_{2}$, and $\mathrm{BaO}$ layers stacked vertically along the $c$-axis of a tetragonal unit cell. Each oxygen atom in the $\mathrm{CuO}_{2}$ plane is shared by two $\mathrm{Cu}$ atoms, resulting in a more direct hopping and a larger bandwidth of the $\mathrm{Cu} d_{x^{2}-y^{2}}$ band compared to that of $\mathrm{Sr}_{2} \mathrm{CuTeO}_{6}$. But the $d_{x^{2}-y^{2}}$ bands of $\mathrm{HgBa}_{2} \mathrm{CuO}_{4}$ are no longer isolated. This requires disentanglement for constructing an effective single-band model.

Like $\mathrm{Sr}_{2} \mathrm{CuTeO}_{6}, \mathrm{HgBa}_{2} \mathrm{CuO}_{4}$ is metallic in DFT, but it is insulating if electron correlations are included as well as in experiment. For all the calculations, we use 845 k-points in the full Brillouin zone; for the DMFT at $\beta=40$, we employ $U=6.5 \mathrm{eV}$. This splits the DFT band into two Hubbard bands (see Fig. 5) and redistributes the k-space occupation of the Wannier orbitals as in the case of $\mathrm{Sr}_{2} \mathrm{CuTeO}_{6}$ [Fig. 2(a)]. The most remarkable difference from that material is the much larger bandwidth in both DFT (white line) and DMFT (color).

Hence, for the very same reason as in the previous section, $\Delta N^{\mathcal{W}}$ has a strong $\mathbf{k}$-dependence. Since we have an effectively two-dimensional model, we plot in Fig. 4(b) $\Delta N^{\mathcal{W}}(\mathbf{k})$ in the plane $k_{z}=0$ of the Brillouin zone. The yellow section of the plane represents the set of $\mathbf{k}$-points that have positive $\Delta N^{\mathcal{W}}$. These states were unoccupied in DFT but get half-occupied in DMFT due to the lower Hubbard band dispersing throughout the Brillouin zone. The negative counterpart (blue) is around the $\Gamma$ point where all states were occupied in DFT. The boundary between these two regions is exactly the DFT Fermi surface marked with a cyan line.

In spite of the fact that both cuprates can be modeled using only a single band, there is a significant correction to the charge density by DMFT. In Fig. 5(b), the correlation-induced charge redistribution $\Delta \rho(\mathbf{r})$ is depicted as an isosurface plot. A negative sign of $\Delta \rho(\mathbf{r})$ (cyan) suggests electron loss from the single band comprised of $\mathrm{Cu} d_{x^{2}-y^{2}}$ and $\mathrm{O} p$ orbitals, a positive sign (yellow) electron gain. There are gains as well as losses around both $\mathrm{Cu}$ and $\mathrm{O}$ sites; altogether some net charge is transferred from $\mathrm{Cu}$ to $\mathrm{O}$.

Let us hence focus on the $\mathrm{O}$ charge gain and $\mathrm{Cu}$ loss in the following. Around the $\mathrm{O}$ sites, the gain has the form of a $p_{x}$ - and $p_{y}$-orbital density pointing to the $\mathrm{Cu}$ sites. It stems from the admixture of these orbitals to our single band. On the $\mathrm{Cu}$ site in turn there are blue $d_{x^{2}-y^{2}}$-like lobes of removed charge pointing toward the neighboring oxygen sites. This indicates that the redistribution $\Delta N^{\mathcal{W}}$ of Wannier orbitals in $\mathbf{k}$-space effectively reduces the level of admixture between these orbitals when moving from the DFT metal to the DMFT insulator. Even though one might naively assume that in a single Wannier band with fixed occupation CSC effects are minor, the density correction is significant for both cuprates.

\section{C. $\mathrm{SrVO}_{3}$}

$\mathrm{SrVO}_{3}$ crystallizes in a cubic perovskite lattice structure and has been the testbed material for DFT+DMFT [33-39], DFT+DCA [40], and GW+DMFT [41-45] method development. A strong interplay between the octahedral crystal field in $\mathrm{VO}_{6}$ and electron correlation determines the properties

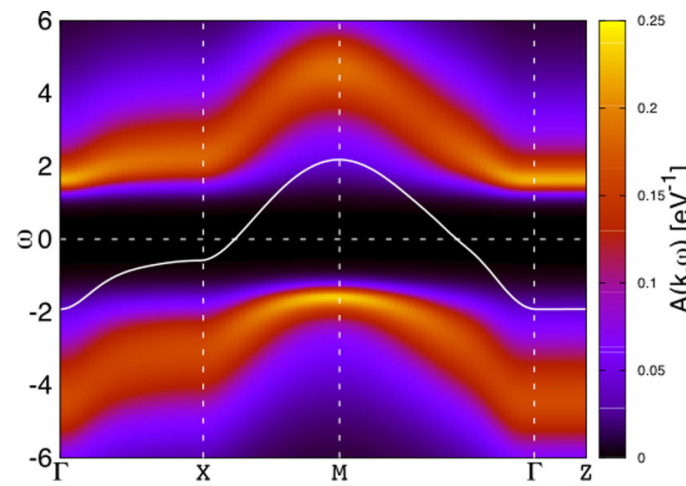

(a)

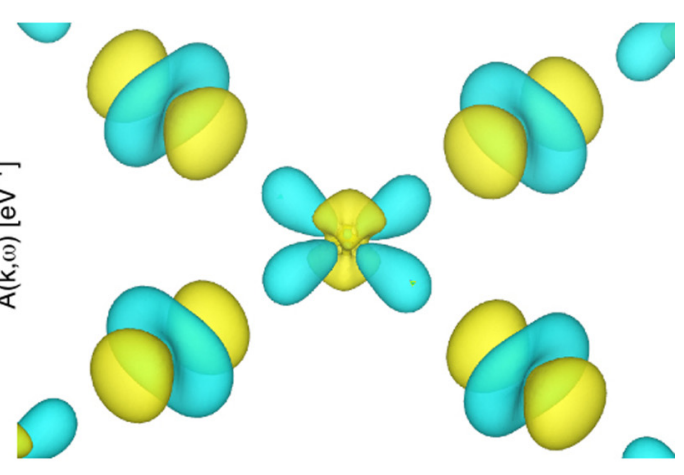

(b)

FIG. 5. (a) k-resolved DMFT spectral function $A(\mathbf{k}, \omega)$ (color) in comparison with the DFT band structure (white line) for $\mathrm{HgBa}_{2} \mathrm{CuO}_{4}$. The dashed horizontal line is the Fermi energy. (b) Isosurface plot of the DMFT charge redistribution $\Delta \rho(\mathbf{r})$. Yellow and cyan correspond to positive and negative values of $\Delta \rho(\mathbf{r})$, at an isovalue of $1.5 \times 10^{-3}$ electrons $\left./ \mathrm{bohr}^{3}\right)$. 


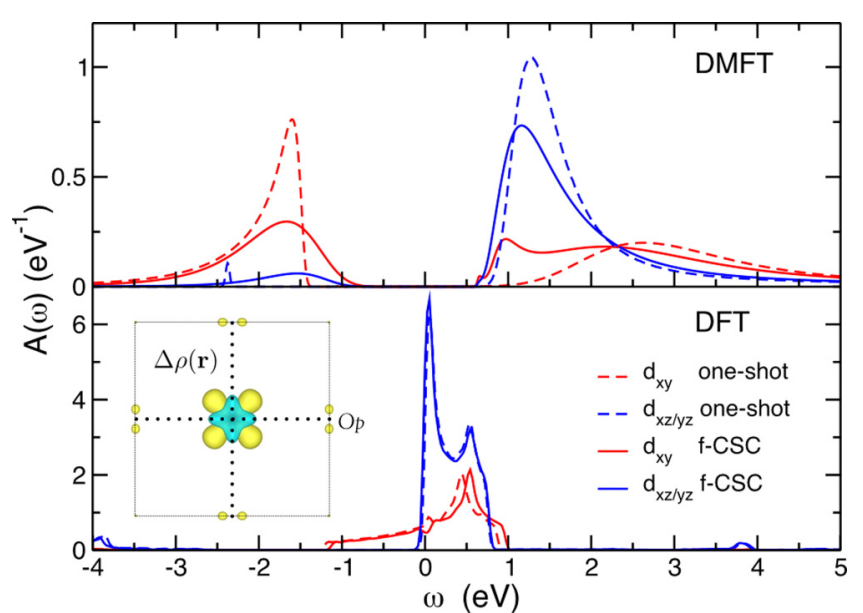

FIG. 6. DMFT (upper panel) and DFT (lower panel) density of states projected onto the $\mathrm{V} t_{2 g}$ orbitals. Dashed lines correspond to calculations without CSC; solid lines are with CSC. Inset: Top view of the isosurface plot of charge redistribution, $\Delta \rho(\mathbf{r})$. Yellow and cyan correspond to positive and negative contributions at an isovalue of $6 \times 10^{-3}$ electrons $/ \mathrm{bohr}^{3}$.

of this material. Bulk $\mathrm{SrVO}_{3}$ exhibits robust metallicity also upon chemical substitution, such as in $\mathrm{Ca}_{1-x} \mathrm{Sr}_{x} \mathrm{VO}_{3}$ [46]. However, the material undergoes a metal-insulator transition if its dimensionality is manipulated [47]. Oxide layers grown on different substrates may, in principle, undergo a charge redistribution at the interface. That is, charge is transferred from one transition-metal ion to that of the neighboring layer. This is, e.g., the case for the $\mathrm{LaTiO}_{3} / \mathrm{LaFeO}_{3}$ interface [48]. However, this is not an issue for $\mathrm{SrTiO}_{3} / \mathrm{SrVO}_{3}$, where the crystal field plays the dominant role. That is, we have a charge redistribution from one orbital to the other [49]. Ultrathin layers (up to three monolayers) of $\mathrm{SrVO}_{3}$ are insulating, which opens the possibility to control the metal-insulator transition by applied electric field or strain, paving the way for a Mott transistor [49].

In ultrathin layers, the bulk $t_{2 g}$ symmetry is broken: the out-of-plane $d_{x z / y z}$ orbitals have a reduced bandwidth, while the in-plane $d_{x y}$ bandwidth remains almost unchanged. Given the $3 d^{1}$ electronic configuration of vanadium, ultrathin layers hence favor the electrons being placed in the $d_{x y}$ orbital. An orbital polarization develops. The orbital reoccupation is quite dramatic in DFT+DMFT: from $1 / 3$ for all $t_{2 g}$ Wannier orbitals in the metallic bulk to almost an occupation of one electron in the $d_{x y}$ orbital for an one-layer film. Let us note that DFT underestimates the orbital polarization, which is strongly enhanced by electronic correlations: in DFT, $d_{x y}$ and $d_{x z / y z}$ orbitals have 0.6 and 0.2 electrons, respectively, for the ultrathin film, and it is metallic. Hence a freestanding monolayer of $\mathrm{SrVO}_{3}$ is ideally suited to study the CSC DFT+DMFT charge redistribution caused by an orbital polarization.

In our calculation, we set the lattice constant ( $a=3.92 \AA)$ to that of a single $\mathrm{SrVO}_{3}$ layer on $\mathrm{SrTiO}_{3}$ as this is the experimental substrate [47]. Figure 6 (lower panel) shows the DFT density of states (DOS), and the dashed lines represent the DOS of the $\mathrm{V} t_{2 g}$ orbitals in DFT. Let us note that the position and width of the $d_{x y}$ band (red) are significantly different compared to that of the $d_{x z / y z}$ bands (blue). This leads to a first tendency toward an orbital polarization already in DFT, giving the aforementioned occupations.

For the DMFT calculations at inverse temperature $\beta=40$, we employ the Kanamori interaction parameters $U^{\prime}=4.0 \mathrm{eV}$, $J=0.75 \mathrm{eV}$ from the literature [49]. The effect of electron correlations in Fig. 6 (upper panel) is twofold: (i) the system becomes a Mott insulator, and (ii) in the insulating phase, $d_{x y}$ is half-filled while the other orbitals are essentially empty. This kind of physics has been observed before [49], but let us now turn to the effect of CSC.

The dashed curves represent the spectral function corresponding to the one-shot DFT+DMFT calculation without CSC, while the solid curves represent the full CSC results. Let us note that the insulating energy gap is slightly reduced by the CSC, which can be attributed to the charge redistribution within the $t_{2 g}$ manifold shown as an inset in Fig. 6. As is to be expected, the charge redistribution has a positive $d_{x y}$-like shape as these orbitals become more occupied. Perpendicular to the plane, shown in the inset, there is a reduced charge in a $d_{x z^{-}}$and $d_{y z}$-like shape.

This changed orbital occupation influences, in turn, the DFT electronic structure; see the solid curves in Fig. 6 (lower panel): The $d_{x y}$ orbital that is more occupied in DMFT is shifted upward to higher energies in DFT and vice versa for $d_{x z / y z}$, as is to be expected already from the Hartree term. That is, DFT partially compensates for the correlation effect of DMFT, but a large net effect remains. This net effect is shown in Fig. 6: the charge redistribution is shown in the inset, and the CSC DFT and DMFT results are shown as solid lines in the main panel.

One-shot DFT+DMFT has an almost filled $d_{x y}$ orbital and almost empty $d_{x z}$ and $d_{y z}$ orbitals, while full CSC results in a slight reduction of the $d_{x y}$-orbital occupancy and vice versa for $d_{x z}$ and $d_{y z}$ orbitals. To emphasize the CSC effect, we have calculated $\Delta \rho^{*}(\mathbf{r})=\Delta \rho^{\text {one-shot }}(\mathbf{r})-\Delta \rho^{\mathrm{CSC}}(\mathbf{r})$, which is depicted in Fig. 7. The positive $d_{x y}$ lobe of $\Delta \rho^{*}(\mathbf{r})$ reflects the reduction in occupancy of that orbital by CSC, and vice versa for the $d_{x z}$ and $d_{y z}$ orbitals. The occupation of $\mathrm{O} p$ orbitals is also reduced significantly. This shows that the effect of full CSC is significant. This is also true for the reduction of the band gap. In the case of the two cuprates (Secs. III A and III B), CSC effects are somewhat less pronounced.

Let us note that all materials considered are essentially two-dimensional so that nonlocal correlations beyond DMFT become important at lower temperatures. These can be described by cluster [50] and diagrammatic [51,52] extensions of DMFT, which yield a k-dependent self-energy. In principle, our approach and formalism can be extended to include such a k-dependent self-energy in the CSC as well.

\section{SUMMARY AND CONCLUSIONS}

We have implemented a fully charge self-consistent DFT+DMFT method, using maximally localized Wannier functions constructed with WANNIER90 [26], the WIEN2K program package, WIEN2WANNIER as an interface, and W2DYNAMICS as an impurity solver. We applied the method 


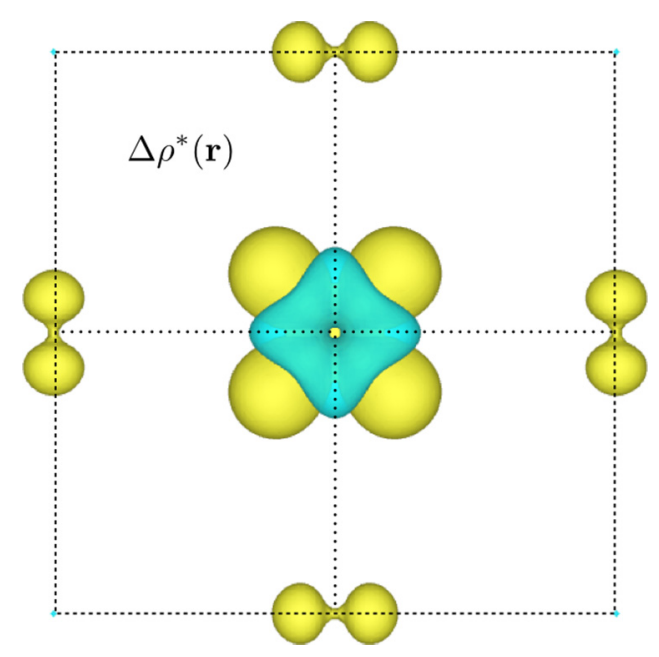

FIG. 7. Top view of the isosurface plot of the difference in charge redistribution, $\Delta \rho^{*}(\mathbf{r})$, over the iterations. Yellow and cyan correspond to positive and negative contributions at an isovalue of $6 \times 10^{-3}$ electrons/bohr ${ }^{3}$.

to strongly correlated electron systems, and we discussed different physical and technical aspects.

The cuprates, $\mathrm{Sr}_{2} \mathrm{CuTeO}_{6}$ and $\mathrm{HgBa}_{2} \mathrm{CuO}_{4}$, can be modeled by a single Wannier orbital. In this situation, one might assume that the charge self-consistency has no effect since this single orbital must remain half-filled; there is no charge redistribution to other orbitals. Nonetheless, the real-space charge density is changed with full CSC DFT+DMFT.

In both cuprates, charge is removed from around the $\mathrm{Cu}$ site and added around the $\mathrm{O}$ sites. Note that oxygen $p$-states are mixed into the single, predominantly $d_{x^{2}-y^{2}}$ orbital. The reason for this change is a change of occupation of the Wannier orbitals in $\mathbf{k}$-space. While for the metallic DFT solution, Wannier functions in some part of the Brillouin zone are singly occupied, in DMFT the band splits into two Hubbard bands and all k-points are occupied equally with half an electron.

Besides this common ground, there are some differences between $\mathrm{Sr}_{2} \mathrm{CuTeO}_{6}$ and $\mathrm{HgBa}_{2} \mathrm{CuO}_{4}$. The former has much weaker $p$ - $d$ hybridization and itinerancy. Hence we do not need disentanglement to Wannier project onto the single orbital. As for the CSC, the changes at the oxygen are much less pronounced because the $\mathrm{O}$ states admix to a much lesser extent in $\mathrm{Sr}_{2} \mathrm{CuTeO}_{6}$. It also has a lower symmetry, which results in a more complicated charge redistribution pattern.

A significant correlation-induced occupation redistribution within the $\mathrm{V} t_{2 g}$ manifold is observed in a single layer of $\mathrm{SrVO}_{3}$. Here, the interplay between crystal field and electron correlation results in a pronounced orbital polarization. The orbital polarization can be clearly identified in the charge redistribution. The CSC has the tendency to counteract the DMFT orbital polarization, which is reduced at self-consistency with respect to one-shot DFT+DMFT.

In all the cases, using single or multiband models, $\Delta N^{\mathcal{W}}(\mathbf{k})$ has a significant $\mathbf{k}$-dependence that translates to an $\mathbf{r}$-dependence of $\Delta \rho(\mathbf{r})$. This shows that there are more profound effects of CSC in DFT+DMFT than the gross effect of charge redistribution from one site to another found in previous studies.

\section{ACKNOWLEDGMENTS}

We thank Patrik Thunström, Rainer Bachleitner, Markus Wallerberger, Oleg Janson, and Peter Blaha for valuable discussions. Financial support from the European Research Council under the European Union's Seventh Framework Program (FP/2007-2013)/ERC through Grant Agreement No. 306447 and by the Austrian Science Fund (FWF) through SFB ViCoM project ID F4103 and I 1395, which is part of the DFG research unit FOR 1346, as well as START project Y746, is gratefully acknowledged. Calculations were done in part on the Vienna Scientific Cluster (VSC).
[1] W. Kohn, Rev. Mod. Phys. 71, 1253 (1999).

[2] R. O. Jones and O. Gunnarsson, Rev. Mod. Phys. 61, 689 (1989).

[3] V. I. Anisimov, F. Aryasetiawan, and A. I. Lichtenstein, J. Phys.: Condens. Matter 9, 767 (1997).

[4] W. Metzner and D. Vollhardt, Phys. Rev. Lett. 62, 324 (1989).

[5] A. Georges and G. Kotliar, Phys. Rev. B 45, 6479 (1992).

[6] A. Georges, G. Kotliar, W. Krauth, and M. Rozenberg, Rev. Mod. Phys. 68, 13 (1996).

[7] V. I. Anisimov, A. I. Poteryaev, M. A. Korotin, A. O. Anokhin, and G. Kotliar, J. Phys.: Condens. Matter 9, 7359 (1997).

[8] A. I. Lichtenstein and M. I. Katsnelson, Phys. Rev. B 57, 6884 (1998).

[9] G. Kotliar, S. Y. Savrasov, K. Haule, V. S. Oudovenko, O. Parcollet, and C. A. Marianetti, Rev. Mod. Phys. 78, 865 (2006).

[10] K. Held, Adv. Phys. 56, 829 (2007).

[11] G. Rohringer, A. Toschi, A. A. Katanin, and K. Held, Phys. Rev. Lett. 107, 256402 (2011).

[12] K. Held, G. Keller, V. Eyert, D. Vollhardt, and V. I. Anisimov, Phys. Rev. Lett. 86, 5345 (2001).
[13] S. Y. Savrasov, G. Kotliar, and E. Abrahams, Nature (London) 410, 793 (2001).

[14] K. Held, A. K. McMahan, and R. T. Scalettar, Phys. Rev. Lett. 87, 276404 (2001).

[15] S. Y. Savrasov and G. Kotliar, Phys. Rev. B 69, 245101 (2004).

[16] J. Minár, L. Chioncel, A. Perlov, H. Ebert, M. I. Katsnelson, and A. I. Lichtenstein, Phys. Rev. B 72, 045125 (2005).

[17] H. Park, A. J. Millis, and C. A. Marianetti, Phys. Rev. B 90, 235103 (2014).

[18] F. Lechermann, A. Georges, A. Poteryaev, S. Biermann, M. Posternak, A. Yamasaki, and O. K. Andersen, Phys. Rev. B 74, 125120 (2006).

[19] L. V. Pourovskii, B. Amadon, S. Biermann, and A. Georges, Phys. Rev. B 76, 235101 (2007).

[20] M. Aichhorn, L. Pourovskii, and A. Georges, Phys. Rev. B 84, 054529 (2011).

[21] M. Aichhorn, L. Pourovskii, V. Vildosola, M. Ferrero, O. Parcollet, T. Miyake, A. Georges, and S. Biermann, Phys. Rev. B 80, 085101 (2009). 
[22] K. Haule, C.-H. Yee, and K. Kim, Phys. Rev. B 81, 195107 (2010).

[23] N. Marzari and D. Vanderbilt, Phys. Rev. B 56, 12847 (1997).

[24] J. Kunes, R. Arita, P. Wissgott, A. Toschi, H. Ikeda, and K. Held, Comput. Phys. Commun. 181, 1888 (2010).

[25] P. Blaha, K. Schwarz, G. K. H. Madsen, D. Kvasnicka, and J. Luitz, WIEN2k, An Augmented Plane Wave + Local Orbitals Program for Calculating Crystal Properties (Karlheinz Schwarz, Techn. Universitat Wien, Austria, 2001).

[26] A. A. Mostofi, J. R. Yates, Y.-S. Lee, I. Souza, D. Vanderbilt, and N. Marzari, Comput. Phys. Commun. 178, 685 (2008).

[27] N. Parragh, A. Toschi, K. Held, and G. Sangiovanni, Phys. Rev. B 86, 155158 (2012); M. Wallerberger et al. (unpublished).

[28] E. Gull, A. J. Mills, A. I. Lichtenstein, A. N. Rubtsov, M. Troyer, and P. Werner, Rev. Mod. Phys. 83, 349 (2011).

[29] J. P. Perdew, J. A. Chevary, S. H. Vosko, K. A. Jackson, M. R. Pederson, D. J. Singh, and C. Fiolhais, Phys. Rev. B 46, 6671 (1992).

[30] T. Koga, N. Kurita, M. Avdeev, S. Danilkin, T. J. Sato, and H. Tanaka, Phys. Rev. B 93, 054426 (2016).

[31] D. Iwanaga, Y. Inaguma, and M. Itoh, J. Solid State Chem. 147, 291 (1999).

[32] S. N. Putilin, E. V. Antipov, O. Chmaissem, and M. Marezio, Nature (London) 362, 226 (1993).

[33] A. Sekiyama, H. Fujiwara, S. Imada, S. Suga, H. Eisaki, S. I. Uchida, K. Takegahara, H. Harima, Y. Saitoh, I. A. Nekrasov, G. Keller, D. E. Kondakov, A. V. Kozhevnikov, Th. Pruschke, K. Held, D. Vollhardt, and V. I. Anisimov, Phys. Rev. Lett. 93, 156402 (2004).

[34] E. Pavarini, S. Biermann, A. Poteryaev, A. I. Lichtenstein, A. Georges, and O. K. Andersen, Phys. Rev. Lett. 92, 176403 (2004).

[35] A. Liebsch, Phys. Rev. Lett. 90, 096401 (2003).

[36] I. A. Nekrasov, G. Keller, D. E. Kondakov, A. V. Kozhevnikov, T. Pruschke, K. Held, D. Vollhardt, and V. I. Anisimov, Phys. Rev. B 72, 155106 (2005).
[37] I. A. Nekrasov, K. Held, G. Keller, D. E. Kondakov, T. Pruschke, M. Kollar, O. K. Andersen, V. I. Anisimov, and D. Vollhardt, Phys. Rev. B 73, 155112 (2006).

[38] Y. Nomura, M. Kaltak, K. Nakamura, C. Taranto, S. Sakai, A. Toschi, R. Arita, K. Held, G. Kresse, and M. Imada, Phys. Rev. B 86, 085117 (2012).

[39] M. Karolak, T. O. Wehling, F. Lechermann, and A. I. Lichtenstein, J. Phys. Condens. Matter 23, 085601 (2011).

[40] H. Lee, K. Foyevtsova, J. Ferber, M. Aichhorn, H. O. Jeschke, and R. Valenti, Phys. Rev. B 85, 165103 (2012).

[41] M. Casula, A. Rubtsov, and S. Biermann, Phys. Rev. B 85, 035115 (2012).

[42] J. M. Tomczak, M. Casula, T. Miyake, F. Aryasetiawan, and S. Biermann, Europhys. Lett. 100, 67001 (2012).

[43] C. Taranto, M. Kaltak, N. Parragh, G. Sangiovanni, G. Kresse, A. Toschi, and K. Held, Phys. Rev. B 88, 165119 (2013).

[44] J. M. Tomczak, M. Casula, T. Miyake, and S. Biermann, Phys. Rev. B 90, 165138 (2014).

[45] S. Choi, A. Kutepov, K. Haule, M. van Schilfgaarde, and G. Kotliar, Quantum Mater. 1, 16001 (2016).

[46] I. H. Inoue, O. Goto, H. Makino, N. E. Hussey, and M. Ishikawa, Phys. Rev. B 58, 4372 (1998).

[47] K. Yoshimatsu, T. Okabe, H. Kumigashira, S. Okamoto, S. Aizaki, A. Fujimori, and M. Oshima, Phys. Rev. Lett. 104, 147601 (2010).

[48] J. E. Kleibeuker, Z. Zhong, H. Nishikawa, J. Gabel, A. Muller, F. Pfaff, M. Sing, K. Held, R. Claessen, G. Koster, and G. Rijnders, Phys. Rev. Lett. 113, 237402 (2014).

[49] Z. Zhong, M. Wallerberger, J. M. Tomczak, C. Taranto, N. Parragh, A. Toschi, G. Sangiovanni, and K. Held, Phys. Rev. Lett. 114, 246401 (2015).

[50] T. Maier, M. Jarrell, T. Pruschke, and M. H. Hettler, Rev. Mod. Phys. 77, 1027 (2005).

[51] A. Toschi, A. A. Katanin, and K. Held, Phys. Rev. B 75, 045118 (2007).

[52] A. N. Rubtsov, M. I. Katsnelson, and A. I. Lichtenstein, Phys. Rev. B 77, 033101 (2008). 